\title{
Bone marrow aspiration complications: Iliopsoas abscess and sacroiliac osteomyelitis
}

\author{
Tuğçe Tural-Kara ${ }^{1}$, Halil Özdemir ${ }^{1}$, Suat Fitöz ${ }^{2}$, Ergin Çiftçi ${ }^{1}$, Fatoş Yalçınkaya ${ }^{3}$ \\ Divisions of ${ }^{1}$ Pediatric Infectious Diseases and ${ }^{3}$ Pediatric Nephrology, Department of Pediatrics, ${ }^{2}$ Department of Pediatric \\ Radiology, Ankara University Faculty of Medicine, Ankara, Turkey.E-mail: tugcetural@hotmail.com \\ Received: 19th April 2016, Revised: 6th May 2016, Accepted 14th July 2016
}

SUMMARY: Tural-Kara T, Özdemir H, Fitöz S, Çiftçi E, Yalçınkaya F. Bone marrow aspiration complications: Iliopsoas abscess and sacroiliac osteomyelitis. Turk J Pediatr 2016; 58: 562-565.

After bone marrow aspiration procedure; some complications like pain and bleeding at the puncture site may be expected but some serious complications like osteomyelitis and soft tissue infections may also rarely occur. In this case we present a boy with recurrent fever. During etiologic investigation, familial Mediterranean fever (FMF) gene M694V mutation was $+/+$. Patient was treated with oral colchicine however fever persisted. The patient was considered as colchicine resistant FMF and steroid treatment was planned. Bone marrow aspiration procedure was executed to rule out malignancy. Three months after bone marrow aspiration, he was readmitted with complaint of left pelvic pain, difficulty in walking without support and standing on his left foot. Radiological imaging demonstrated left iliopsoas abscess and left sacroiliac osteomyelitis. Patient was successfully treated with intravenous ampicillin-sulbactam and clindamycin treatment for 6 weeks. Then oral amoxicillin-clavulanic acid treatment was continued for 2 weeks. Patient was discharged without any surgical procedure. On 1-year follow-up he could walk without any support.

Key words: bone marrow aspiration, children, iliopsoas abscess, sacroiliac osteomyelitis.

Iliopsoas abscess and sacroiliac osteomyelitis are rarely seen in children. History of pelvic trauma is reported in some cases as a predisposing factor. It is difficult to diagnose these diseases due to deep localization of infection site and lack of specific signs and symptoms ${ }^{1-3}$.

Bone marrow aspiration is commonly used for diagnosis of various hematological disorders, assessment of prognosis and treatment response ${ }^{4}$. The most common bone marrow aspiration site is posterior superior iliac crest. After aspiration pelvic pain and bleeding at the puncture site are reported frequently, but bone marrow aspiration may also cause some rare complications ${ }^{5}$. We report a boy with iliopsoas abscess and sacroiliac osteomyelitis which are unusual complications of bone marrow aspiration.

\section{Case Report}

An 18-month-old boy was admitted to our hospital with a complaint of fever that lasted 3 to 4 days and was not responsive to antipyretic treatment. He had recurrent fever 1-2 times per month since he was 1-monthold. He received antibiotics several times with no benefit. There were no abnormal finding in physical examination. Laboratory tests for recurrent fever etiology were normal except familial Mediterranean fever (FMF) gene mutation which was $\mathrm{M} 694 \mathrm{~V}+/+$. Oral colchicine therapy was given but fever continued despite of this therapy. Patient was considered as colchicine resistant FMF and steroid treatment was planned. He was consulted to Pediatric Oncology Department for bone marrow aspiration procedure to rule out malignancy before steroid treatment. Puncture was performed from left posterior superior iliac spine. Cytology of bone marrow aspiration material was normal. No pathogens were isolated from the aspiration material.

Three months after the bone marrow aspiration, 
he was readmitted to hospital with complaints of left pelvic pain, difficulty in walking without support and standing on his left foot. These complaints began within 3-4 days after the bone marrow aspiration and during this period intermittent fever continued. On physical examination, limited range of left pelvic joint motion, pain with movements (abduction, adduction, internal rotation) of left leg, tenderness and pain at the puncture site was observed but neurological examination was intact. Laboratory examination revealed: hemoglobin count of $11.3 \mathrm{~g} / \mathrm{dl}$, leukocyte count of $20,500 / \mathrm{mm}^{3}$ (neutrophils $68 \%$, lymphocytes $25 \%$ and monocytes $7 \%$ ), platelet count of $536,000 / \mathrm{mm}^{3}$, erythrocyte sedimentation rate of $64 \mathrm{~mm} / \mathrm{h}$ and C-reactive protein concentration of $14 \mathrm{mg} / \mathrm{dl}$. Pelvic computed tomography (CT) showed left iliopsoas abscess near the iliopsoas muscle with many enlarged lymph nodes, increased density and soft tissue in left sacroiliac joint and in L5-S2 neural foramen, lytic destructive changes in sacrum and ilium (Fig. 1). Sacroiliac joint magnetic resonance imaging (MRI) showed T2 signal enhancement near bony structures and left sacroiliac joint, contrast enhancement areas, cortical thickening and periosteal reaction in sacroiliac bone (osteomyelitis), contrast enhancement in S1-2 and in S2-3 spinal roots, signal and contrast enhancement of left iliacus muscle and left iliac lymph nodes (Fig. 2A, 2B). Patient was diagnosed with sacroiliac osteomyelitis and iliopsoas abscess.

Percutaneous abscess drainage was performed and methicillin sensitive Staphylococcus aureus was isolated from drainage culture material. Intravenous ampicillin-sulbactam and clindamycin treatment were given for 6 weeks. During follow-up fever disappeared and clinical findings improved. In addition, the pain on left foot reduced and he started to stand on his foot without support. Levels of acute phase reactants decreased under the treatment. After discharge, oral amoxicillin-clavulanic acid treatment was continued for 2 weeks per orally. Patient did not have any complaints and he could walk without support.

\section{Discussion}

Iliopsoas abscess (IPA) is an unusual disease in children. It is identified as primary and secondary IPA due to etiology. Primary IPA is more frequently seen in children and it has hematogenous or lymphatic origin because of the rich vascularity of iliopsoas muscle. Secondary IPA occurs directly by spreading of pathogens from the contagious infection site such as pyelonephritis, osteomyelitis, appendicitis and others ${ }^{1,2}$. The most causative pathogen is $S$. aureus ${ }^{6,7}$.

Sacroiliac osteomyelitis is also rare in children. Patients may present with different clinical signs. The most common complaints are fever and pain at the infected area ${ }^{3}$. Although diagnosis is difficult, ultrasonography and pelvic $\mathrm{CT}$ are useful imaging. The most common causative microorganism which is isolated from drainage material is S. aureus $(80 \%)$.

Bone marrow aspiration is necessary for diagnosis of various diseases. It helps complete hematologic evaluation. It is useful for diagnosis of fever with unknown origin, storage diseases, infiltrative disease and malignancy. However, some complications may be seen after bone marrow aspiration. Myeloproliferative disorders, treatment with aspirin or warfarin, obesity and disseminated intravascular coagulation are some risk factors leading to complications. In literature some complications such as prolonged pain, loss of function, bleeding, hematoma, needle breakage, bone fracture, compartment syndrome and infections were reported after bone marrow aspiration and biopsy $^{8-12}$. Bleeding has been reported as the most common complication ${ }^{12}$.

In Bain et al.8's study between 1995 and 2001,

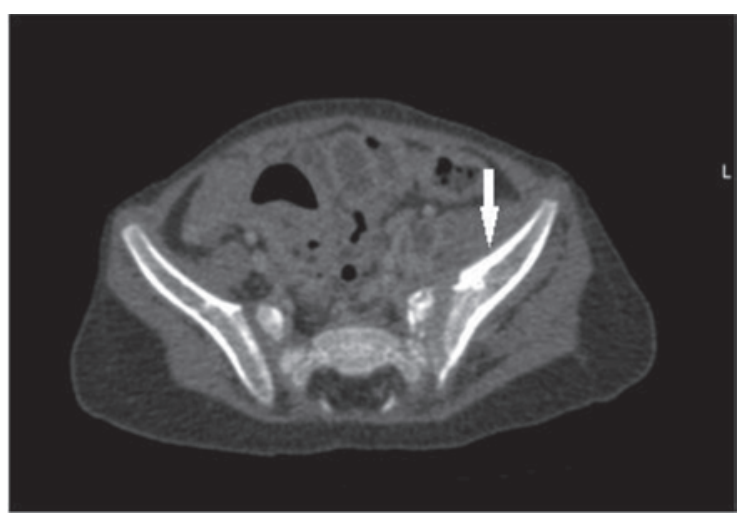

Fig. 1. Axial CT images of pelvis reveal enlargement of left sacroiliac joint and irregular bone margins. The iliopsoas muscle is thickened and heterogeneous. More caudal image shows necrotic lymph nodes. 

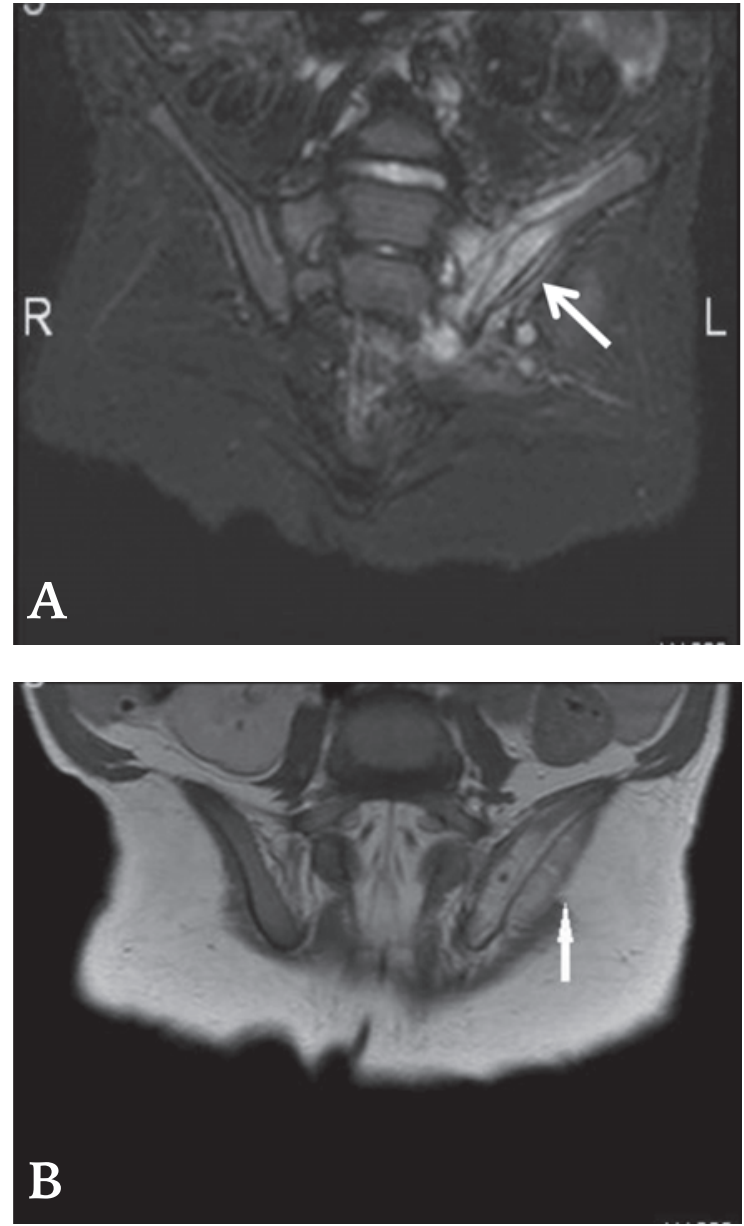

Fig. 2. Oblique coronal (2A) STIR MR images demonstrate hyperintense bone marrow changes associated with soft tissue indicating osteomyelitis. Also note subperiosteal abscess of the left iliac bone (arrow). Contrast-enhanced oblique coronal MR T1-weighted image (2B) shows heterogeneous enhancement of the left iliac bone.

54,890 bone marrow biopsies were performed and adverse events occurred in 26 of them. The most common complication was hemorrhage which was reported in 14 patients. Thirteen patients with complication of bleeding had hematologic-oncologic diseases. Needle related incidents were detected in 7 patients and surgical removal was needed in 3 of them. Infections such as minor superficial infection, abscess and cellulitis were seen in 3 patients. In the same center 20,323 bone marrow aspiration and biopsies were performed in 2004 and 15 adverse events were reported such as hemorrhage $(n=9)$, persistent pain $(n=3)$, collapse relating to previous undiagnosed severe aortic stenosis $(n=1)$, anaphylactic reaction $(n=1)$ and fracture at the biopsy site $(n=1)^{9}$.
Salem et al. ${ }^{10}$ reported two adult patients with hematoma which was secondary to bone marrow biopsy. Both patients recovered without sequelae. Berber et al. ${ }^{11}$ detected arteriovenous fistula (AVF) in a 76-year-old man after bone marrow aspiration and biopsy. They suggested that in cases with refractory bleeding AVF should be considered.

To our knowledge, this is the first case which reports occurrence of iliopsoas abscess and sacroiliac osteomyelitis after bone marrow aspiration. Iliopsoas abscess and sacroiliac osteomyelitis were considered as bone marrow aspiration complications, but it was not possible to accept as a definite cause. Our patient had fever with unknown etiology and bone marrow aspiration was performed to determine the focus of fever. There is limited data about infection related complications after bone marrow aspiration. In only one case report in the literature, a 24-year-old adult patient with sternal osteomyelitis which occurred at the puncture site was reported. In this case, $S$. aureus was isolated from the lesion culture. The patient was successfully treated with intravenous nafcillin and oral dicloxacillin ${ }^{13}$.

In conclusion; bone marrow aspiration may cause some rare complications. If patients have unusual complaints, unusual complications such as iliopsoas abscess and sacroiliac osteomyelitis should be considered as a complication of bone marrow aspiration and biopsy. Therefore, advanced imaging should be performed.

\section{REFERENCES}

1. Shields D, Robinson P, Crowley TP. Iliopsoas abscess--a review and update on the literature. Int J Surg 2012; 10: 466-469.

2. Breese JS, Edward MS. Psoas abscess in children. Pediatr Infect Dis J 1990; 9: 201-206.

3. Klein JD, Leach KA. Pediatric pelvic osteomyelitis. Clin Pediatr 2007; 46: 787-790.

4. Hsu CH, Tsai TY, Ma HP. Retroperitoneal hematoma after bone marrow aspiration in a polycythemia vera patient. J Exp Clin Med 2013; 5: 156-157.

5. Gladden K, Spill GR. Iliac fracture after a bone marrow biopsy. PM R 2011; 3: 1150-1152.

6. Malhotra R, Singh KD, Bhan S, Dave PK. Primary pyogenic abscess of the psoas muscle. J Bone Joint Surg Am 1992; 74: 278-284.

7. Shinhar D, Mares AJ, Finaly R, Cohen Z, Barki Y. Psoas abscess: Diagnostic dilemma in childhood. Harefuah 1996; 131: 402-404. 
8. Bain BJ. Bone marrow biopsy morbidity and mortality. Br J Haematol 2003; 121: 949-951.

9. Bain BJ. Morbidity associated with bone marrow aspiration and trephine biopsy - a review of UK data for 2004. Haematologica 2006; 91: 1293-1294.

10. Salem P, Wolverson MK, Reimers HJ, Kudva GC. Complications of bone marrow biopsy. Br J Haematol 2003; 121: 821 .
11. Berber I, Erkurt MA, Kuku I, et al. An unexpected complication of bone marrow aspiration and trephine biopsy: Arteriovenous fistula. Med Princ Pract 2014; 23: $380-383$.

12. Berumen-Nafarrate E, Vega-Najera C, Leal-Contreras C, Leal-Berumen I. Gluteal compartment syndrome following an iliac bone marrow aspiration. Case Rep Orthop 2013; 2013: 812172.

13. Shah M, Watanakunakorn C. Staphylococcus aureus sternal osteomyelitis complicating bone marrow aspiration. South Med J 1978; 71: 348-349. 